\title{
Selecting a Regression Saturated by Indicators
}

Hendry, David F.; Johansen, Søren; Santos, Carlos

Publication date:

2007

Document version

Publisher's PDF, also known as Version of record

Citation for published version (APA):

Hendry, D. F., Johansen, S., \& Santos, C. (2007). Selecting a Regression Saturated by Indicators. Department of Economics, University of Copenhagen. 


\section{Discussion Papers Department of Economics University of Copenhagen}

No. $07-26$

Selecting a Regression Saturated by Indicators

David F. Hendry, Søren J ohansen, and Carlos Santos

Studiestræde 6, DK-1455 Copenhagen K., Denmark

Tel.: +45353230 82 - Fax: +453532 3000

http://www.econ.ku.dk

ISSN: 1601-2461 (online) 


\title{
Selecting a Regression Saturated by Indicators
}

\author{
David F. Hendry, Economics Department, Oxford University \\ Søren Johansen, Economics Department, University of Copenhagen \\ and CREATES University of Aarhus \\ and Carlos Santos, Department of Economics and Management, \\ Portuguese Catholic University, Porto*
}

November 7, 2007

\begin{abstract}
We consider selecting a regression model, using a variant of Gets, when there are more variables than observations, in the special case that the variables are impulse dummies (indicators) for every observation. We show that the setting is unproblematic if tackled appropriately, and obtain the finite-sample distribution of estimators of the mean and variance in a simple location-scale model under the null that no impulses matter. A Monte Carlo simulation confirms the null distribution, and shows power against an alternative of interest.
\end{abstract}

JEL classifications: C51, C22.

Key words: Indicators; regression saturation; subset selection; model selection.

\section{Introduction}

We consider the application of automatic general-to-specific (Gets) model selection procedures when there are more variables $m$ than observations $N$ in the special case that a model is saturated with a complete set of $N$ impulse indicators, one for every observation. In this setting, the initial general unrestricted model (GUM) cannot be estimated at the outset. Instead, Hendry and Krolzig (2004) propose 'subset selection' by PcGets across combinations of candidate variables, each search path leading to a terminal model, followed by searches across the union of these. ${ }^{1}$ We show that their approach can be applied successfully to the selection of indicators. For general analyses of Gets, see inter alia Hoover and Perez (1999, 2004), Krolzig and Hendry (2001), Hendry and Krolzig (2003, 2005), Campos, Hendry and Krolzig (2003), Granger and Hendry (2005), and Campos, Ericsson and Hendry (2004); details of the standard algorithm in PcGets are presented in the appendix to Hendry and Krolzig (2001).

When $m>N$, all regressors cannot be entered simultaneously. Consequently, models based on combinations of subsets of $m_{1} \leq N / 2$ variables are explored seriatim, and a new joint model is formulated from all the terminal models thereby selected. If this union model is sufficiently small, PcGets can be applied as usual; otherwise repeated serial searches are required. Variants of this algorithm are discussed by Hendry and Krolzig (2004). Under the null that none of the $N$ indicator variables (impulses) matters, we derive the distributions of post-selection estimators of the mean and variance in a simple location-scale data generation process (DGP). A Monte Carlo simulation confirms the null distributions obtained, and shows power against a range of alternatives of practical interest in econometrics. We also show that

${ }^{*}$ Financial support from the UK Economic and Social Research Council under a professorial Research Fellowship, RES 051 270035 and grant RES 000 230539; support from Center for Research in Econometric Analysis of Time Series, CREATES, funded by the Danish National Research Foundation; and funding from the Fundação para Ciência e a Tecnologia (Lisboa) are gratefully acknowledged by the first, second and the third author respectively.

${ }^{1}$ PcGets is an Ox Package (see Doornok 1999) implementing automatic general-to-specific (Gets) modelling for linear regression models based on the theory of reduction (see Hendry 1995, Ch.9). 
exploring many combinations of subsets of indicators does not affect the null rejection frequency of the procedure, but could be advantageous under the alternative that breaks have occurred. Finally, noting that any regressor can be expressed as an exact function of $N$ impulse indicators, we explore the more general case of $K>N$ candidate regressor variables when in fact $k<<N$ are relevant.

As an analogy, the PcGets search procedure attempts to sieve valuable information (regressors that genuinely matter) from 'garbage' (regressors that are in fact irrelevant, but this is not known to the investigator). Its properties when doing so for $m<<N$ are described in Hendry and Krolzig (2005). The sieving can be achieved in one step in that case, namely all candidate regressors are added $a b$ initio, and checked for relevance by multi-path searches, using critical values that depend on $m, N$, and the investigator's perceived costs of over, versus under, selection. If the total set of candidates exceeds the sieve's capacity, the search is conducted in stages, designed to ensure that almost all loworder interactions between regressors are examined. Here we establish the sampling properties under the null when $m=N+1$ candidate variables are postulated, and interpret the outcomes. Other approaches to $m>N$ include e.g., Foster and Stine (2004).

The paper is organized as follows. Section 2 considers model selection when there are too many indicators for the available sample. Section 3 derives the mean and variance of the sampling distribution of the mean, and section 4 presents simulation evidence on its finite-sample accuracy and the power of the procedure to detect some forms of location shift. Section 6 concludes.

\section{Model selection with $N$ indicator variables}

We consider the behaviour for regressions which are 'saturated' by indicator variables. Let an observed random variable $y_{i}$ be independently normally distributed as $y_{i} \sim \operatorname{IN~}\left[\mu, \sigma_{\varepsilon}^{2}\right]$ for $i=1, \ldots, N$, where $\mu \in \mathcal{R}, \sigma_{\varepsilon}^{2} \in \mathcal{R}_{+}$are the parameters of interest. However, an investigator is uncertain where outliers (if any) may lurk. She therefore defines a saturating set of $N$ indicators $d_{j, i}=1_{\{j=i\}}$, one for every $j$, and wishes to estimate $\mu$ and $\sigma_{\varepsilon}^{2}$ from a regression of $y_{i}$ on $\left\{\mu, d_{j, i}, j=1, \ldots, N-1\right\}$. Since a perfect fit will always result from such a regression, nothing is learned.

As a first step, consider instead adding half of the indicators (e.g., $d_{j, i}$ for $j=1, \ldots, N / 2$, assuming for simplicity that $N$ is even) together with the intercept. Thus we consider the general unrestricted (GUM) of the first step:

$$
y_{i}=\mu+\sum_{j=1}^{N / 2} \delta_{j} d_{j, i}+\varepsilon_{i} .
$$

Hence, (1) contains $N / 2$ parameters for $N / 2$ impulse indicators for the first $N / 2$ observations, as well as the mean and variance. Below, we consider alternative divisions of the indicators across the sample.

We find:

$$
\begin{aligned}
\widehat{\mu}_{1} & =\frac{1}{N / 2} \sum_{i=N / 2+1}^{N} y_{i}, \\
s_{1}^{2} & =\frac{1}{N / 2-1} \sum_{i=N / 2+1}^{N}\left(y_{i}-\widehat{\mu}_{1}\right)^{2} \\
\widehat{\delta}_{i} & =y_{i}-\widehat{\mu}_{1}, \quad i=1, \ldots, N / 2
\end{aligned}
$$

so that:

$$
\begin{aligned}
\widehat{\varepsilon}_{i} & =0, i=1, \ldots, N / 2 \\
\widehat{\varepsilon}_{i} & =y_{i}-\widehat{\mu}_{1}, \quad i=N / 2+1, \ldots, N
\end{aligned}
$$


Because the estimates of $\mu$ and $\sigma^{2}$ are the usual ones for the remaining sample, we find that:

$$
\mathrm{E}\left[\widehat{\mu}_{1}\right]=\mu \text { and } \operatorname{Var}\left[\widehat{\mu}_{1}\right]=(N / 2)^{-1} \sigma_{\varepsilon}^{2},
$$

and:

$$
\mathrm{E}\left[s_{1}^{2}\right]=\sigma_{\varepsilon}^{2} .
$$

Consequently, both GUM estimators are unbiased at this stage.

Next, adopting the usual PcGets approach, a parsimonious model is selected from (1) such that all mis-specification tests remain insignificant and all retained variables are significant at the desired level. That terminal model is stored, ensuring the intercept is one of the 'variables' retained by assigning it a fixed status. This selection simply involves eliminating any indicator where $\left|\mathrm{t}_{1, \widehat{\delta}_{i}}\right|<c_{\alpha}$, when the significance level $c_{\alpha}$ is used (such as that corresponding to $\alpha=0.025$ or $\alpha=0.01$ or more generally, a function of $N$ to control the false retention rate under the null).

Now re-commence from the equivalent of (1), but entering only the other half of the impulses namely $\left(1, d_{i, j}, j=N / 2+1, \ldots, N\right)$, repeat the process to estimate $\mu$ and $\sigma^{2}$ by $\widehat{\mu}_{2}$ and $s_{2}^{2}$, then again apply PcGets, eliminating indicators where $\left|\mathrm{t}_{2, \widehat{\delta}_{i}}\right|<c_{\alpha}$ and storing the resulting parsimonious selection. Lastly, formulate a model where all significant selected indicators from the two terminal models are combined, and re-select from that for the final model. This demonstrates that despite saturating by indicators, a feasible algorithm exists for checking every observation.

The final estimates are:

$$
\widetilde{\mu}=\frac{\sum_{i=1}^{N_{1}} y_{i} 1_{\left\{\left|t_{1, \widehat{\delta}_{i}}\right|<c_{\alpha}\right\}}+\sum_{i=N_{1}+1}^{N} y_{i} 1_{\left\{\left|t_{2, \widehat{\delta}_{i}}\right|<c_{\alpha}\right\}}}{\sum_{i=1}^{N_{1}} 1_{\left\{\left|t_{1, \hat{\delta}_{i}}\right|<c_{\alpha}\right\}}+\sum_{i=N_{1}+1}^{N} 1_{\left\{\left|t_{2, \widehat{\delta}_{i}}\right|<c_{\alpha}\right\}}}
$$

and

$$
\tilde{\sigma}_{\varepsilon}^{2}=\frac{\sum_{i=1}^{N_{1}}\left(y_{i}-\widehat{\mu}_{1}\right)^{2} 1_{\left\{\left|t_{1, \widehat{\delta}_{i}}\right|<c_{\alpha}\right\}}+\sum_{i=N_{1}+1}^{N}\left(y_{i}-\widehat{\mu}_{2}\right)^{2} 1_{\left\{\left|t_{2, \hat{\delta}_{i}}\right|<c_{\alpha}\right\}}}{\sum_{i=1}^{N_{1}} 1_{\left\{\left|t_{1, \widehat{\delta}_{i}}\right|<c_{\alpha}\right\}}+\sum_{i=N_{1}+1}^{N} 1_{\left\{\left|t_{2, \widehat{\delta}_{i}}\right|<c_{\alpha}\right\}}-1} .
$$

The next section presents a formal analysis and derives the asymptotic properties of the estimators (5) and (6).

Although the 'perfect fit' problem no longer arises, it may be thought that the huge number of $N / 2$ indicators entered in each stage might induce spurious significance. However, the corresponding group of observations is simply 'dummied out' for estimating $\mu$, which is then just the mean of the remaining sample. For an approximately normal distribution, $\alpha N$ outliers will occur on average under the null for a significance level $\alpha$, so $\alpha N / 2$ indicators will be selected on average at each stage, and $\alpha N$ overall: an indicator will be significant at level $\alpha$ if and only if there is an $\alpha$-level outlier at that observation. Under the null, therefore, the proposed procedure is close to finding outliers relative to the whole sample mean $\widehat{\mu}$ and variance $\widehat{\sigma}^{2}$ : nevertheless, under some alternatives, the procedure can yield very different outcomes from (say) direct comparison with a criterion, such as being greater than $2 \widehat{\sigma}$ in absolute value, as figure 3 below illustrates.

Additional regressors will entail an inability to add half the indicators at each stage, and may necessitate exploring many combinations, but do not otherwise affect the analysis. More generally, to ensure adequate power against reasonable alternatives, many-way divisions could be used to check that breaks do not occur at any precise division point (such as $N / 2$ ), as discussed in sub-section 5 , and checked by simulation in section 4 .

Conversely, testing many different forms of hypothesis could alter the null rejection frequency. For example, checking the joint significance of all possible pairs, triplets, etc. will not deliver a null rejection frequency of $\alpha$. This is not a serious issue under the null hypothesis that only $\delta_{i}=0$ for all $i$; but 
researchers may have a temptation to consider (e.g.) step shifts where blocks of $\delta_{i}$ take the same values. To control the null rejection frequency, the number of classes of hypotheses has to be controlled, and one way of achieving that goal is to restrict such hypothesis searches to situations where the null has been rejected. Conditional on that occurrence, then many alternatives of how to form an index of the retained indicators can be entertained, which will not affect the null rejection frequency: Hendry and Santos (2005) show that after selecting indicators, indexes thereof can be formed without distorting inference.

There is a selection effect on the mean and variance estimates in the final model, similar to 'trimming', and the approximate distributions are derived in section 3. The 3-stage PcGets procedure is difficult to analyze directly, so the approach therein is to eliminate half of the sample by adding half the indicators (see Salkever (1976)), then select outliers in the remainding half. Next, the converse halfsample is removed and the other group of outliers detected. This procedure entails that on both steps, all outliers in the saturated half are also removed, so is close to the third stage of PcGets. The analysis then derives the distribution of the mean based on the two subsample means, as well as the mean of the error variance. In fact, since an exact sample split is not needed, and may sometimes be undesirable, the analysis allows for a general split, and in section 3.3 considers the possibility that many splits are used.

The role of the Monte Carlo experiments in section 4 is, therefore, to check that the theory is indeed closely relevant to the PcGets procedure in small samples when the null distribution is a standard normal, as well as being relevant for other distributions.

\section{Sampling distributions}

We first derive the sampling distribution of $\widetilde{\mu}$ under the null after dummy saturation, then consider the impact of saturation on $\widetilde{\sigma}_{\varepsilon}^{2}$.

\subsection{Asymptotic distributions of $\widetilde{\mu}$}

We derive the asymptotic distribution of $\widetilde{\mu}$ calculated under the assumptions that the first analysis has $N_{1}$ dummies and the second has $N_{2}=N-N_{1}$ dummies, whereas the data generating process has IID variables.

Theorem 1 Let $y_{1}, \ldots, y_{N}$ be IID with a symmetric continuous density $f(\cdot)$ with mean $\mu$ and $\mathrm{E}\left(y_{i}^{8}\right)<$ $\infty$. Let $N=N_{1}+N_{2}$, and assume that $N_{1} / N \rightarrow \lambda_{1}$ and $N_{2} / N \rightarrow \lambda_{2}$ where $0<\lambda_{1}, \lambda_{2}<1$, with $\lambda_{1}+\lambda_{2}=1$, then the limit distribution of the estimator $\widetilde{\mu}$, see (5), is given by:

$$
N^{1 / 2}(\widetilde{\mu}-\mu) \rightarrow \mathrm{N}\left[0, \sigma_{\varepsilon}^{2} \sigma_{\mu}^{2}\right]
$$

where

$$
\sigma_{\mu}^{2}=\left(\int_{-c_{\alpha}}^{c_{\alpha}} f(\varepsilon) d \varepsilon\right)^{-2}\left[\int_{-c_{\alpha}}^{c_{\alpha}} \varepsilon^{2} f(\varepsilon) d \varepsilon\left(1+4 c_{\alpha} f\left(c_{\alpha}\right)\right)+\left(\frac{\lambda_{1}^{2}}{\lambda_{2}}+\frac{\lambda_{2}^{2}}{\lambda_{1}}\right)\left(2 c_{\alpha} f\left(c_{\alpha}\right)\right)^{2}\right] .
$$

Note that $\int_{-c_{\alpha}}^{c_{\alpha}} f(\varepsilon) d \varepsilon=1-\alpha$, and for the normal distribution, $f(\varepsilon)=\frac{1}{\sigma_{\varepsilon}} \phi\left(\frac{\varepsilon}{\sigma_{\varepsilon}}\right)$, we find the expression:

$$
\int_{-c_{\alpha}}^{c_{\alpha}} \varepsilon^{2} \phi(\varepsilon) d \varepsilon=\int_{-c_{\alpha}}^{c_{\alpha}} \phi(\varepsilon) d \varepsilon-2 c_{\alpha} \phi\left(c_{\alpha}\right)
$$

so that under normality for an equal split $\left(\lambda_{1}=\lambda_{2}\right.$ :

$$
\sigma_{\mu}^{2}=\frac{1}{(1-\alpha)}\left(1+4 c_{\alpha} \phi\left(c_{\alpha}\right)-\frac{2 c_{\alpha} \phi\left(c_{\alpha}\right)}{(1-\alpha)}\left[1+2 c_{\alpha} \phi\left(c_{\alpha}\right)\right]\right) .
$$


Proof. The is no loss of generality in setting $\sigma_{\varepsilon}^{2}=1$, and we let $c=c_{\alpha}$. The estimator satisfies:

$$
N^{1 / 2}(\widetilde{\mu}-\mu)=\frac{N^{-1 / 2}\left(\sum_{i=1}^{N_{1}} \varepsilon_{i} 1_{\left\{\left|\varepsilon_{i}-\bar{\varepsilon}_{1}\right| \leq c s_{1} \sqrt{1+N_{2}^{-1}}\right\}}+\sum_{i=N_{1}+1}^{N} \varepsilon_{i} 1_{\left\{\left|\varepsilon_{i}-\bar{\varepsilon}_{2}\right| \leq c s_{2} \sqrt{1+N_{1}^{-1}}\right\}}\right)}{N^{-1}\left(\sum_{i=1}^{N_{1}} 1_{\left\{\left|\varepsilon_{i}-\bar{\varepsilon}_{1}\right| \leq c s_{1} \sqrt{1+N_{2}^{-1}}\right\}}+\sum_{i=N_{1}+1}^{N} 1_{\left\{\left|\varepsilon_{i}-\bar{\varepsilon}_{2}\right| \leq c s_{2} \sqrt{1+N_{1}^{-1}}\right\}}\right)}=\frac{B_{N}}{M_{N}} .
$$

We show that $B_{N}$ converges in distribution to a normal distribution, and $M_{N}$ converges in probability to a constant. The problem is the dependence structure due to the appearance of $\left(\bar{\varepsilon}_{1}, s_{1}^{2}\right)$ and $\left(\bar{\varepsilon}_{2}, s_{2}^{2}\right)$ in the selection variables. We therefore define the simpler variables which are sums of IID variables:

$$
\begin{aligned}
K_{N} & =N^{-1}\left(\sum_{i=1}^{N_{1}} 1_{\left\{\left|\varepsilon_{i}\right| \leq c\right\}}+\sum_{i=N_{1}+1}^{N} 1_{\left\{\left|\varepsilon_{i}\right| \leq c\right\}}\right) \\
C_{N} & =N^{-1 / 2}\left(\sum_{i=1}^{N_{1}}\left(\varepsilon_{i} 1_{\left\{\left|\varepsilon_{i}\right| \leq c\right\}}+2 c f(c) \bar{\varepsilon}_{1}\right)+\sum_{i=N_{1}+1}^{N}\left(\varepsilon_{i} 1_{\left\{\left|\varepsilon_{i}\right| \leq c\right\}}+2 c f(c) \bar{\varepsilon}_{2}\right)\right) .
\end{aligned}
$$

We want to approximate $B_{N} / M_{N}$ by $C_{N} / K_{N}$ and so write:

$$
N^{1 / 2}(\widetilde{\mu}-\mu)=\frac{B_{N}}{M_{N}}=\frac{\left(B_{N}-C_{N}\right)+C_{N}}{\left(M_{N}-K_{N}\right)+K_{N}} .
$$

From the law of large numbers:

$$
K_{N} \stackrel{P}{\rightarrow} \int_{-c}^{c} f(\varepsilon) d \varepsilon
$$

By symmetry of the distribution, $\mathrm{E}\left[C_{N}\right]=0$, and from:

$$
C_{N}=N^{-1 / 2}\left(\sum_{i=1}^{N_{1}}\left(\varepsilon_{i} 1_{\left\{\left|\varepsilon_{i}\right| \leq c\right\}}+\frac{\lambda_{2}}{\lambda_{1}} 2 c f(c) \varepsilon_{i}\right)+\sum_{i=N_{1}+1}^{N}\left(\varepsilon_{i} 1_{\left\{\left|\varepsilon_{i}\right| \leq c\right\}}+\frac{\lambda_{1}}{\lambda_{2}} 2 c f(c) \varepsilon_{i}\right)\right)
$$

so from the central limit theorem, $C_{N}$ is asymptotically normal with mean zero and variance:

$$
\begin{aligned}
& \lambda_{1}\left[\mathrm{E}\left[\varepsilon^{2} 1_{\{|\varepsilon| \leq c\}}\right]+\left(\frac{\lambda_{2}}{\lambda_{1}}\right)^{2}(2 c f(c))^{2}+4 c f(c) \frac{\lambda_{2}}{\lambda_{1}} \mathrm{E}\left[\varepsilon^{2} 1_{\{|\varepsilon| \leq c\}}\right]\right] \\
& +\lambda_{2}\left[\mathrm{E}\left[\varepsilon^{2} 1_{\{|\varepsilon| \leq c\}}\right]+\left(\frac{\lambda_{1}}{\lambda_{2}}\right)^{2}(2 c f(c))^{2}+4 c f(c) \frac{\lambda_{1}}{\lambda_{2}} \mathrm{E}\left[\varepsilon^{2} 1_{\{|\varepsilon| \leq c\}}\right]\right] \\
= & \mathrm{E}\left[\varepsilon^{2} 1_{\{|\varepsilon| \leq c\}}\right](1+4 c f(c))+\left(\frac{\lambda_{2}^{2}}{\lambda_{1}}+\frac{\lambda_{1}^{2}}{\lambda_{2}}\right)(2 c f(c))^{2},
\end{aligned}
$$

which together with (9) gives is the expression for $\sigma_{\mu}^{2}$. We therefore only have to prove that:

$$
\begin{aligned}
& M_{N}-K_{N} \stackrel{\mathrm{P}}{\rightarrow} 0, \\
& B_{N}-C_{N} \stackrel{\mathrm{P}}{\rightarrow} 0 .
\end{aligned}
$$

To prove (10) we note that it is enough to show that:

$$
D_{N}=N_{1}^{-1} \sum_{i=1}^{N_{1}}\left(1_{\left\{\left|\varepsilon_{i}-\bar{\varepsilon}_{1}\right| \leq c s_{1} \sqrt{1+N_{2}^{-1}}\right\}}-1_{\left\{\left|\varepsilon_{i}\right| \leq c\right\}}\right) \stackrel{\mathrm{P}}{\rightarrow} 0
$$


since the other one follows by replacing subscript 1 by 2 . Let $u=\bar{\varepsilon}_{1}$ and $v=c\left(s_{1} \sqrt{1+N_{2}^{-1}}-1\right)$ and apply the inequality:

$$
\left|1_{\left\{\left|\varepsilon_{i}-\bar{\varepsilon}_{1}\right| \leq c s_{1} \sqrt{1+N_{2}^{-1}}\right\}}-1_{\left\{\mid \varepsilon_{i} \leq c\right\}}\right|=\left|1_{\left\{\left|\varepsilon_{i}-u\right| \leq c+v\right\}}-1_{\left\{\left|\varepsilon_{i}\right| \leq c\right\}}\right| \leq 1_{\left\{\left|\varepsilon_{i}-c\right| \leq|u|+|v|\right\}}+1_{\left\{\left|\varepsilon_{i}+c\right| \leq|u|+|v|\right\}}
$$

to find:

$$
N_{1}^{-1} \mathrm{E}_{u v}\left|D_{N}\right| \leq \int_{c-|u|-|v|}^{c+|u|+|v|} \varepsilon f(\varepsilon) d \varepsilon+\int_{-c-|u|-|v|}^{-c+|u|+|v|} \varepsilon f(\varepsilon) d \varepsilon=h(|u|+|v|),
$$

which is bounded and continuous in $|u|+|v|$ by the assumptions. Because $|u|+|v| \stackrel{\mathrm{P}}{\rightarrow} 0$, we then get, by taking expectations, that:

$$
N_{1}^{-1} \mathrm{E}\left|D_{N}\right| \leq \mathrm{E}[h(|u|+|v|)] \rightarrow h(0)=0 .
$$

This shows that $D_{N} \stackrel{\mathrm{P}}{\rightarrow} 0$ and hence (10).

We next prove (11). It is enough to show that:

$$
R_{N}=N_{1}^{-1 / 2} \sum_{i=1}^{N_{1}}\left(\varepsilon_{i} 1_{\left\{\left|\varepsilon_{i}-\bar{\varepsilon}_{1}\right| \leq c s_{1} \sqrt{1+N_{2}^{-1}}\right\}}-\varepsilon_{i} 1_{\left\{\left|\varepsilon_{i}\right| \leq c\right\}}-2 c f(c) \bar{\varepsilon}_{1}\right) \stackrel{\mathrm{P}}{\rightarrow} 0 .
$$

By symmetry, we have that $\mathrm{E}\left[R_{N}\right]=0$, and we want to show that $\operatorname{Var}\left[R_{N}\right] \rightarrow 0$.

To find the variance, we again condition on $\bar{\varepsilon}_{1}=u$ and $c\left(s_{1} \sqrt{1+N_{2}^{-1}}-1\right)=v$, which are independent of the variables $\varepsilon_{1}, \ldots, \varepsilon_{N_{1}}$, which remain IID, and find:

$$
\begin{aligned}
\mathrm{E}_{u v}\left[R_{N}\right] & =N_{1}^{1 / 2} \mathrm{E}\left[\varepsilon_{i} 1_{\left\{\left|\varepsilon_{i}-u\right| \leq c+v\right\}}-\varepsilon_{i} 1_{\left\{\left|\varepsilon_{i}\right| \leq c\right\}}-2 c f(c) u\right] \\
& =N_{1}^{1 / 2}\left(\int_{-c-v+u}^{c+v+u} \varepsilon f(\varepsilon) d \varepsilon-\int_{-c}^{c} \varepsilon f(\varepsilon) d \varepsilon-2 c f(c) u\right) .
\end{aligned}
$$

From Taylor's formula with remainder term, we find for a differentiable function:

$$
g(c+h)=g(c)+h g\left(c^{*}\right)=g(c)+h g(c)+h\left(g\left(c^{*}\right)-g(c)\right),\left|c-c^{*}\right| \leq|h| .
$$

This implies that, using $f(c)=f(-c)$ :

$$
\begin{aligned}
\int_{-\infty}^{c+v+u} \varepsilon f(\varepsilon) d \varepsilon & =\int_{-\infty}^{c} \varepsilon f(\varepsilon) d \varepsilon+(u+v) c f(c)+(u+v)\left(c^{*} f\left(c^{*}\right)-c f(c)\right), \\
\int_{-\infty}^{-c-v+u} \varepsilon f(\varepsilon) d \varepsilon & =\int_{-\infty}^{-c} \varepsilon f(\varepsilon) d \varepsilon-(u-v) c f(c)+(u-v)\left(-c^{* *} f\left(c^{* *}\right)+c f(c)\right) .
\end{aligned}
$$

Subtracting these expressions, we find that:

$$
\left|\mathrm{E}_{u v}\left[R_{N}\right]\right| \leq N_{1}^{1 / 2}(|u|+|v|)\left(\left|c^{*} f\left(c^{*}\right)-c f(c)\right|+\left|c^{* *} f\left(c^{* *}\right)-c f(c)\right|\right) .
$$

Hence:

$$
\begin{aligned}
\operatorname{Var}\left(\mathrm{E}_{u v}\left[R_{N}\right]\right) & \left.\leq \mathrm{E}\left(\mathrm{E}_{u v} R_{N}\right]\right)^{2} \\
& \leq N_{1} \mathrm{E}(|u|+|v|)^{2}\left(\left|c^{*} f\left(c^{*}\right)-c f(c)\right|+\left|c^{* *} f\left(c^{* *}\right)-c f(c)\right|\right)^{2} \\
& \leq 2 N_{1} \mathrm{E}\left(u^{2}+v^{2}\right)\left(\left|c^{*} f\left(c^{*}\right)-c f(c)\right|+\left|c^{* *} f\left(c^{* *}\right)-c f(c)\right|\right)^{2} \\
& \leq 2^{3 / 2} N_{1}\left(\mathrm{E}\left(u^{4}+v^{4}\right)\right)^{1 / 2} E\left(\left(\left|c^{*} f\left(c^{*}\right)-c f(c)\right|+\left|c^{* *} f\left(c^{* *}\right)-c f(c)\right|\right)^{4}\right)^{1 / 2}
\end{aligned}
$$


where we used the inequality $(a+b)^{2} \leq 2\left(a^{2}+b^{2}\right)$ twice and the Cauchy-Schwartz inequality to separate the expectations.

Note that because $\left|\varepsilon_{i}\right|$ has a finite mean, we have $|c| f(c) \rightarrow 0,|c| \rightarrow \infty$, so that the continuity of $f(\cdot)$ implies $|c| f(c)$ is a bounded continuous function. Because

$$
\max \left(\left|c-c^{* *}\right|,\left|c-c^{*}\right|\right) \leq|u|+|v|=\left|\bar{\varepsilon}_{1}\right|+c\left|s_{1} \sqrt{1+N_{2}^{-1}}-1\right| \stackrel{\mathrm{P}}{\rightarrow} 0,
$$

it follows that $c^{*} \stackrel{\mathrm{P}}{\rightarrow} c$ and $c^{* *} \stackrel{\mathrm{P}}{\rightarrow} c$, so that:

$$
E\left(\left(\left|c^{*} f\left(c^{*}\right)-c f(c)\right|+\left|c^{* *} f\left(c^{* *}\right)-c f(c)\right|\right)^{4}\right) c^{*} \rightarrow 0 .
$$

We then have to prove that $N_{1}^{2} \mathrm{E}\left(u^{4}+v^{4}\right)$ is bounded. The first term is

$$
N_{1}^{2} \mathrm{E}\left(\bar{\varepsilon}_{1}^{4}\right)=N_{1}^{-1} E\left(\varepsilon_{1}^{4}\right)+3\left(1-N_{1}^{-1}\right),
$$

using that $E\left(\varepsilon_{1}\right)=E\left(\varepsilon_{1}^{3}\right)=0$ and $E\left(\varepsilon_{1}^{2}\right)=1$. This is bounded when we assume finite fourth moment. Next:

$$
N_{1}^{2} \mathrm{E}\left(s_{1} \sqrt{1+N_{2}^{-1}}-1\right)^{4} \leq 8\left[N_{1}^{2} \mathrm{E}\left(s_{1}-1\right)^{4}\left(1+N_{2}^{-1}\right)^{2}+N_{1}^{2}\left(1-\sqrt{1+N_{2}^{-1}}\right)^{4}\right]
$$

The factor $\left(1+N_{2}^{-1}\right)^{2}$ and the term $N_{1}^{2}\left(1-\sqrt{1+N_{2}^{-1}}\right)^{4}$ are bounded, and we evaluate:

$$
\begin{aligned}
N_{1}^{2} \mathrm{E}\left(s_{1}-1\right)^{4} & \leq N_{1}^{2} \mathrm{E}\left(s_{1}^{2}-1\right)^{4} \\
& =N_{1}^{-1} E\left(\varepsilon_{t}^{2}-1\right)^{4}+3\left(1-N_{1}^{-1}\right)\left(E\left(\varepsilon_{t}^{2}-1\right)^{2}\right)^{2},
\end{aligned}
$$

which is bounded when $\varepsilon_{t}$ has moments of order eight. Thus the first factor $N_{1}^{2} \mathrm{E}\left(u^{4}+v^{4}\right)$ is bounded and therefore:

$$
\operatorname{Var}\left(\mathrm{E}_{u v}\left[R_{N}\right]\right) \rightarrow 0 .
$$

Next we consider $\mathrm{E}\left[\operatorname{Var}_{u v}\left(R_{N}\right)\right]$ and find using the inequality (13) that:

$$
\begin{aligned}
\operatorname{Var}_{u v}\left(R_{N}\right) & =\mathrm{E}\left[\varepsilon_{1} 1_{\left\{\left|\varepsilon_{1}-u\right| \leq c+v\right\}}-\varepsilon_{1} 1_{\left\{\left|\varepsilon_{1}\right| \leq c\right\}}\right]^{2} \\
& \leq \int_{-c-|u|-|v|}^{-c+|u|+|v|} \varepsilon^{2} f(\varepsilon) d \varepsilon+\int_{c-|u|-|v|}^{c+|u|+|v|} \varepsilon^{2} f(\varepsilon) d \varepsilon
\end{aligned}
$$

which is a bounded continuous function of $|u|+|v|$, so that:

$$
\mathrm{E}\left[\operatorname{Var}_{u v}\left(R_{N}\right)\right] \rightarrow 0
$$

Combining (14) and (16) we see that $\operatorname{Var}\left(R_{N}\right) \rightarrow 0$, which completes the proof of (11).

\subsection{The probability limit of $\widetilde{\sigma}_{\varepsilon}^{2}$}

Theorem 2 Under the assumptions of Theorem 1 it holds that the estimator $\widetilde{\sigma}_{\varepsilon}^{2}$, see (6), has the limit:

$$
\tilde{\sigma}_{\varepsilon}^{2} \stackrel{P}{\rightarrow} \frac{\int_{-c_{\alpha}}^{c_{\alpha}} \varepsilon^{2} f(\varepsilon) d \varepsilon}{\int_{-c_{\alpha}}^{c_{\alpha}} f(\varepsilon) d \varepsilon}=\operatorname{Var}\left(\varepsilon|| \varepsilon \mid<c_{\alpha}\right) .
$$


For the normal distribution, $f(\varepsilon)=\frac{1}{\sigma_{\varepsilon}} \phi\left(\frac{\varepsilon}{\sigma_{\varepsilon}}\right)$, we find the expression:

$$
\frac{\int_{-c_{\alpha}}^{c_{\alpha}} \varepsilon^{2} \phi(\varepsilon) d \varepsilon}{\int_{-c_{\alpha}}^{c_{\alpha}} \phi(\varepsilon) d \varepsilon}=\sigma_{\varepsilon}^{2}\left(1-\frac{2 c_{\alpha} \phi\left(c_{\alpha}\right)}{1-\alpha}\right) .
$$

Proof. The technique is the same as in the proof of Theorem 1 . We let $\sigma_{\varepsilon}^{2}=1$, and let $c=c_{\alpha}$. We first note that, see (6), $\widetilde{\sigma}_{\varepsilon}^{2}=\frac{D_{N}}{K_{N}}+H_{N}$, where:

$$
\begin{aligned}
\frac{D_{N}}{K_{N}} & =\frac{N^{-1} \sum_{i=1}^{N_{1}} \varepsilon_{i}^{2} 1_{\left\{\left|\varepsilon_{i}-\widehat{\varepsilon}_{1}\right| \leq c s_{1} \sqrt{1+N_{2}^{-1}}\right\}}+\sum_{i=N_{1}+1}^{N} \varepsilon_{i}^{2} 1_{\left\{\left|\varepsilon_{i}-\widehat{\varepsilon}_{2}\right| \leq c s_{2} \sqrt{1+N_{1}^{-1}}\right\}}}{N^{-1} \sum_{i=1}^{N_{1}} 1_{\left\{\left|\varepsilon_{i}-\widehat{\varepsilon}_{1}\right| \leq c s_{1} \sqrt{1+N_{2}^{-1}}\right\}}+\sum_{i=N_{1}+1}^{N} 1_{\left\{\left|\varepsilon_{i}-\widehat{\varepsilon}_{2}\right| \leq c s_{2} \sqrt{1+N_{1}^{-1}}\right\}}}, \\
H_{N} & =\frac{\left(\mu-\widehat{\mu}_{1}\right)^{2} \sum_{i=1}^{N_{1}} 1_{\left\{\left|\varepsilon_{i}-\widehat{\varepsilon}_{1}\right| \leq c s_{1} \sqrt{1+N_{2}^{-1}}\right\}}+\left(\mu-\widehat{\mu}_{2}\right)^{2} \sum_{i=N_{1}+1}^{N} 1_{\left\{\left|\varepsilon_{i}-\widehat{\varepsilon}_{2}\right| \leq c s_{2} \sqrt{1+N_{1}^{-1}}\right\}}}{\sum_{i=1}^{N_{1}} 1_{\left\{\left|\varepsilon_{i}-\widehat{\varepsilon}_{1}\right| \leq c s_{1} \sqrt{1+N_{2}^{-1}}\right\}}+\sum_{i=N_{1}+1}^{N} 1_{\left\{\left|\varepsilon_{i}-\widehat{\varepsilon}_{2}\right| \leq c s_{2} \sqrt{1+N_{1}^{-1}}\right\}}} .
\end{aligned}
$$

The last term, $H_{N}$, tends to zero in probability because $\widehat{\mu}_{1} \stackrel{\mathrm{P}}{\rightarrow} \mu$ and $\widehat{\mu}_{2} \stackrel{\mathrm{P}}{\rightarrow} \mu$.

From (9), we know that $K_{N} \stackrel{\mathrm{P}}{\rightarrow} \int_{-c}^{c} f(\varepsilon) d \varepsilon$. We define the sum of independent variables and apply the law of large numbers to find::

$$
E_{N}=N^{-1}\left(\sum_{i=1}^{N_{1}} \varepsilon_{i}^{2} 1_{\left\{\left|\varepsilon_{i}\right| \leq c\right\}}+\sum_{i=N_{1}+1}^{N} \varepsilon_{i}^{2} 1_{\left\{\left|\varepsilon_{i}\right| \leq c\right\}}\right) \stackrel{\mathrm{P}}{\rightarrow} \int_{-c}^{c} \varepsilon^{2} f(\varepsilon) d \varepsilon .
$$

We next have to show that $E_{N}-D_{N} \stackrel{\mathrm{P}}{\rightarrow} 0$. It is clearly enough to prove that:

$$
N_{1}^{-1} \sum_{i=1}^{N_{1}} \varepsilon_{i}^{2}\left(1_{\left\{\left|\varepsilon_{i}-\widehat{\varepsilon}_{1}\right| \leq c s_{1} \sqrt{1+N_{2}^{-1}}\right\}}-1_{\left\{\mid \varepsilon_{i} \leq c\right\}}\right) \stackrel{\mathrm{P}}{\rightarrow} 0 .
$$

Conditioning on $u$ and $v$ we find using (13) that:

$$
\begin{aligned}
& E_{u v}\left|N_{1}^{-1} \sum_{i=1}^{N_{1}} \varepsilon_{i}^{2}\left(1_{\left\{\left|\varepsilon_{i}-\widehat{\varepsilon}_{1}\right| \leq c s_{1} \sqrt{\left.1+N_{2}^{-1}\right\}}\right.}-1_{\left\{\mid \varepsilon_{i} \leq c\right\}}\right)\right| \\
\leq & E\left[\varepsilon_{1}^{2}\left(1_{\left\{\left|\varepsilon_{1}-c\right| \leq|u|+|v|\right\}}+1_{\left\{\left|\varepsilon_{1}+c\right| \leq|u|+|v|\right\}}\right]\right. \\
\leq & \int_{c-|u|-|v|}^{c+|u|+|v|} \varepsilon^{2} f(\varepsilon) d \varepsilon+\int_{-c-|u|-|v|}^{-c+|u|+|v|} \varepsilon^{2} f(\varepsilon) d \varepsilon,
\end{aligned}
$$

see (15). This is a bounded and continuous function of $|u|+|v|$ and hence the expectation tends to zero.

\subsection{Many splits}

We split the data into $I_{j}, j=1, \ldots, m$ with $N_{j}=\lambda_{j} N$ elements and estimators $\bar{y}_{j}, s_{j}^{2}$ and define

$$
\begin{aligned}
N_{-j} & =\sum_{k \neq j} N_{k}=N-N_{j}, \lambda_{-j}=1-\lambda_{j} \\
\bar{y}_{-j} & =\frac{\sum_{i \notin I_{j}} y_{i}}{\sum_{i \notin I_{j}} 1}=\frac{\sum_{k \neq j} N_{k} \bar{y}_{k}}{\sum_{k \neq j} N_{k}} \\
s_{-j}^{2} & =\frac{\sum_{k \neq j}\left(N_{k}-1\right) s_{k}^{2}}{\sum_{k \neq j}\left(N_{k}-1\right)}
\end{aligned}
$$




$$
\tilde{\mu}=\frac{\sum_{j=1}^{m} \sum_{i \in I_{j}} y_{i} 1_{\left\{\left|y_{i}-\bar{y}_{-j}\right|<c_{\alpha} s_{-j} \sqrt{1+N_{-j}^{-1}}\right\}}}{\sum_{j=1}^{m} \sum_{i \in I_{j}} 1_{\left\{\left|y_{i}-\bar{y}_{-j}\right|<c_{\alpha} s_{-j} \sqrt{1+N_{-j}^{-1}}\right\}}}
$$

and

$$
\tilde{\sigma}_{\varepsilon}^{2}=\frac{\sum_{j=1}^{m} \sum_{i \in I_{j}}\left(y_{i}-\bar{y}_{-j}\right)^{2} 1_{\left\{\left|y_{i}-\bar{y}_{-j}\right|<c_{\alpha} s_{-j} \sqrt{1+N_{-j}^{-1}}\right\}}}{\sum_{j=1}^{m} \sum_{i \in I_{j}} 1_{\left\{\left|y_{i}-\bar{y}_{-j}\right|<c_{\alpha} s_{-j} \sqrt{1+N_{-j}^{-1}}\right\}}} .
$$

\subsection{Asymptotic distributions of $\widetilde{\mu}$ and limit of $\widetilde{\sigma}_{\varepsilon}^{2}$.}

Theorem 3 Let $y_{1}, \ldots, y_{N}$ be IID with a symmetric continuous density $f(\cdot)$ with mean $\mu$ and $\mathrm{E}\left(y_{i}^{8}\right)<$ $\infty$. Let $N=\sum_{j=1}^{m} N_{j}$, and assume that $N_{j} / N \rightarrow \lambda_{j}$, where $0<\lambda_{j}<1$, with $\sum_{j=1}^{m} \lambda_{j}=1$, then the limit distribution of the estimator $\tilde{\mu}$, see (17), is given by:

$$
N^{1 / 2}(\widetilde{\mu}-\mu) \rightarrow \mathrm{N}\left[0, \sigma_{\varepsilon}^{2} \sigma_{\mu}^{2}\right]
$$

where

$$
\sigma_{\mu}^{2}=\left(\int_{-c_{\alpha}}^{c_{\alpha}} f(\varepsilon) d \varepsilon\right)^{-2} \int_{-c_{\alpha}}^{c_{\alpha}} \varepsilon^{2} f(\varepsilon) d \varepsilon\left(1+4 c_{\alpha} f\left(c_{\alpha}\right)\right)+\sum_{j=1}^{m} \lambda_{j}\left[\sum_{k \neq j} \frac{\lambda_{k}}{1-\lambda_{k}}\right]^{2}\left(2 c_{\alpha} f\left(c_{\alpha}\right)\right)^{2}
$$

If in particular $N_{1}=\ldots=N_{m}$, then $\sum_{j=1}^{m} \lambda_{j}\left[\sum_{k \neq j} \frac{\lambda_{k}}{\lambda_{-k}}\right]^{2}=1$.

Proof. There is no loss of generality in setting $\sigma_{\varepsilon}^{2}=1$, and we let $c=c_{\alpha}$. The estimator satisfies:

$$
N^{1 / 2}(\widetilde{\mu}-\mu)=\frac{\left.N^{-1 / 2} \sum_{j=1}^{m} \sum_{i \in I_{j}} \varepsilon_{i} 1_{\left\{\left|\varepsilon_{i}-\bar{\varepsilon}_{-j}\right|<c_{\alpha} s_{-j}\right.} \sqrt{1+N_{-j}^{-1}}\right\}}{N^{-1} \sum_{j=1}^{m} \sum_{i \in I_{j}} 1_{\left\{\left|\varepsilon_{i}-\bar{\varepsilon}_{-j}\right|<c_{\alpha} s_{-j} \sqrt{1+N_{-j}^{-1}}\right\}}}=\frac{B_{N}}{M_{N}}
$$

We show that $B_{N}$ converges in distribution to a normal distribution, and $M_{N}$ converges in probability to a constant. The problem is the dependence structure due to the appearance of $\left(\bar{\varepsilon}_{-j}, s_{-j}^{2}\right)$ in the selection variables. We therefore define the simpler variables which are sums of IID variables:

$$
\begin{aligned}
K_{N} & =N^{-1} \sum_{j=1}^{m} \sum_{i \in I_{j}} 1_{\left\{\left|\varepsilon_{i}\right|<c_{\alpha}\right\}} \\
C_{N} & =N^{-1 / 2} \sum_{j=1}^{m} \sum_{i \in I_{j}}\left(\varepsilon_{i} 1_{\left\{\left|\varepsilon_{i}\right| \leq c\right\}}+2 c f(c) \bar{\varepsilon}_{-j}\right) .
\end{aligned}
$$

We want to approximate $B_{N} / M_{N}$ by $C_{N} / K_{N}$ and so write:

$$
N^{1 / 2}(\widetilde{\mu}-\mu)=\frac{B_{N}}{M_{N}}=\frac{\left(B_{N}-C_{N}\right)+C_{N}}{\left(M_{N}-K_{N}\right)+K_{N}} .
$$

From the law of large numbers:

$$
K_{N} \stackrel{P}{\rightarrow} \int_{-c}^{c} f(\varepsilon) d \varepsilon
$$


By symmetry of the distribution, $\mathrm{E}\left[C_{N}\right]=0$, and from:

$$
\begin{aligned}
C_{N} & =N^{-1 / 2} \sum_{j=1}^{m} \sum_{i \in I_{j}}\left(\varepsilon_{i} 1_{\left\{\left|\varepsilon_{i}\right| \leq c\right\}}+2 c f(c) \bar{\varepsilon}_{-j}\right) \\
& =N^{-1 / 2} \sum_{j=1}^{m} \sum_{i \in I_{j}}\left(\varepsilon_{i} 1_{\left\{\left|\varepsilon_{i}\right| \leq c\right\}}+2 c f(c) \varepsilon_{i}\left[\sum_{k \neq j} \frac{N_{k}}{N_{-k}}\right]\right)
\end{aligned}
$$

so from the central limit theorem, $C_{N}$ is asymptotically normal with mean zero and variance:

$$
\begin{aligned}
& N^{-1} \sum_{j=1}^{m} N_{j} \mathrm{E}\left[\varepsilon^{2} 1_{\{|\varepsilon| \leq c\}}\right]+\left[\sum_{k \neq j} \frac{N_{k}}{N_{-k}}\right]^{2}(2 c f(c))^{2}+4 c f(c)\left[\sum_{k \neq j} \frac{N_{k}}{N_{-k}}\right] \mathrm{E}\left[\varepsilon^{2} 1_{\{|\varepsilon| \leq c\}}\right] \\
= & \mathrm{E}\left[\varepsilon^{2} 1_{\{|\varepsilon| \leq c\}}\right]\left(1+4 c f(c) N^{-1} \sum_{j=1}^{m} N_{j} \sum_{k \neq j} \frac{N_{k}}{N_{-k}}\right)+N^{-1} \sum_{j=1}^{m} N_{j}\left[\sum_{k \neq j} \frac{N_{k}}{N_{-k}}\right]^{2}(2 c f(c))^{2} \\
= & \mathrm{E}\left[\varepsilon^{2} 1_{\{|\varepsilon| \leq c\}}\right]\left(1+4 c f(c) \sum_{j=1}^{m} \lambda_{j} \sum_{k \neq j} \frac{\lambda_{k}}{\lambda_{-k}}\right)+\sum_{j=1}^{m} \lambda_{j}\left[\sum_{k \neq j} \frac{\lambda_{k}}{\lambda_{-k}}\right]^{2}(2 c f(c))^{2} .
\end{aligned}
$$

next we show that

$$
\sum_{j=1}^{m} \lambda_{j} \sum_{k \neq j} \frac{\lambda_{k}}{\lambda_{-k}}=\sum_{k \neq j} \frac{\lambda_{j} \lambda_{k}}{1-\lambda_{k}}=\sum_{k=1}^{m} \sum_{j \neq k} \frac{\lambda_{j} \lambda_{k}}{1-\lambda_{k}}=\sum_{k=1}^{m} \frac{\left(1-\lambda_{k}\right) \lambda_{k}}{1-\lambda_{k}}=1
$$

which together with (9) gives is the expression for $\sigma_{\mu}^{2}$.

If in particular $\lambda_{i}=m^{-1}$, then

$$
\sum_{j=1}^{m} \lambda_{j}\left[\sum_{k \neq j} \frac{\lambda_{k}}{\lambda_{-k}}\right]^{2}=\sum_{j=1}^{m} \frac{1}{m}\left[\sum_{k \neq j} \frac{\frac{1}{m}}{1-\frac{1}{m}}\right]^{2}=\sum_{j=1}^{m} \frac{1}{m}\left[(m-1) \frac{1}{m-1}\right]^{2}=1 .
$$

\section{Monte Carlo experiments}

We first examine the properties of the retained impulses under normality, checking that the PcGets procedure delivers retention rates which closely match the binomial expansion of $(\alpha+[1-\alpha])^{N}$ despite the sequential selection. Next, we check that using three equal-sized $N / 3$ sample splits does not affect the null outcome. Then we investigate the empirical distribution of $\widetilde{\sigma}_{\varepsilon}^{2}$ under the null, before turning to that of $\widetilde{\mu}$ to check the small-sample accuracy of the derivations in section 3 . We also briefly consider the impact of saturation in the highly non-normal case of a t(4)-distributed error. Finally, we consider some empirical rejection frequencies of the saturation procedure under two simple alternatives.

We consider a simple location-scale DGP:

$$
y_{i}=\mu+\varepsilon_{i}
$$

with:

$$
\varepsilon_{i} \sim \operatorname{IN}\left[0, \sigma_{\varepsilon}\right] .
$$


In the simulations, we will set $\mu=0$ and $\sigma_{\varepsilon}=1$. The aim is to investigate the impact on estimating $\mu$ and $\sigma_{\varepsilon}^{2}$ when saturating the model with impulse dummies.

We consider two econometric models. The first is given by:

$$
y_{i}=\mu+\sum_{j=1}^{N-N / 2} \delta_{j} d_{i, j}+\varepsilon_{i}
$$

whilst the second is:

$$
y_{i}=\mu+\sum_{i=N / 2+1}^{N} \delta_{j} d_{i, j}+\varepsilon_{i}
$$

$N$ is the sample size and $d_{i, j}$ is a single impulse indicator. Hence, (24) contains $N / 2$ parameters for $N / 2$ impulse indicators for the first $N / 2$ observations; and (25) contains $N / 2$ impulse indicators for the last set of observations. Below, we consider alternative divisions of the indicators across the sample.

\subsection{Empirical rejection frequencies of impulse indicators under the normal null}

Given the DGP, the composite null hypothesis:

$$
\mathrm{H}_{0}: \delta_{i}=0 \forall i
$$

is true, $\forall i$, for both models. We first estimate model (24) and then model (25) in that order, under these assumptions, store the significant indicators, and combine these to obtain the final selected model, and hence estimators akin to (5) and (6). $M=10,000$ replications were conducted for this experiment. From Hendry and Santos (2005), the OLS estimators of $\delta_{i}$ are unbiased and tests of (26) have Student $\mathrm{t}_{(N-N / 2)}$ distributions under the null. Table 1 reports the mean rejection frequency (RF) of the null for a sample of 50 observations at nominal rejection frequencies per test of 5\%,2.5\% and $1 \%$. The empirical rejection frequencies are close to the nominals.

\begin{tabular}{|c|c|c|}
\hline Mean $\mathrm{RF}_{5 \%}$ & Mean $\mathrm{RF}_{2.5 \%}$ & Mean $\mathrm{RF}_{1 \%}$ \\
\hline 0.0499 & 0.0250 & 0.0101 \\
\hline
\end{tabular}

Table 1: Rejection frequencies of impulse indicators in (22)

This outcome is not affected by randomly, rather than consecutively, adding $N / 2$ dummies in each regression, unsurprisingly since the data have no time ordering. Under an alternative where the break is a location shift, such shuffling could be useful, as we show below.

\subsubsection{Empirical distributions of retained impulses}

Under the null hypothesis, the distributions of the numbers of empirically retained impulses are of interest: retention is decided on the basis of a two-sided individual significance test. We report these for $N=50$ and $N=100$ using the above settings, but including additional significance levels.

The first plot refers to $N=50$ and uses a two-sided t-test with a $1 \%$ significance level. The $x$ axis measures the number of impulses retained, and the $y$-axis the actual number of regressions (out of $10,000)$ that retained the given number of 'spurious' impulses.

The mode occurs at zero with probability $(1-\alpha)^{N} \simeq 0.6$, with the probability of retaining one impulse by chance being $N \alpha \times(1-\alpha)^{N-1} \simeq 0.3$. As figure 1a also shows, a three-way equal split of $N / 3$ does not change the outcomes substantively: neither the mode nor the decay pattern alters. Corresponding outcomes held at nominal sizes of $2.5 \%$ and $5 \%$. 



Figure 1: Distributions of impulses, means and equation standard errors for $\alpha=1 \%$

Figure $1 \mathrm{~b}$ records the impact on the mean null rejection frequency of using finer equal sub-divisions of added impulses at $N=50$ for $\alpha=0.01$, so $\alpha N=0.5$. There is little change in rejection frequency as the number of equal splits increases, especially given that the uncertainty bars are $\pm 2 \times 0.005$ (one standard error bars are shown for the first two splits). The overall range of the mean estimate is 0.490 to 0.496 , so there is in fact slight under selection.

\subsection{Empirical distribution of $\widetilde{\mu}$ under the null}

Figure 1c shows the empirical distributions of $\widetilde{\mu}$ and $\widehat{\mu}$ under the null for $N=100$. Throughout, we use $\widehat{\mu}$ and $\widehat{\sigma}_{\varepsilon}^{2}$ as the full-sample OLS estimators of the mean and variance. $\widetilde{\mu}$ and $\widetilde{\sigma}_{\varepsilon}^{2}$ are the estimators for the impulse saturated model. The distribution of $\widehat{\mu}$ is correctly centered, and more concentrated near the center, but as shown above, more dispersed in the tails, leading to a larger standard deviation.

\subsection{Empirical distribution of $\widetilde{\sigma}_{\varepsilon}^{2}$ under the normal null}

Figure $1 \mathrm{~d}$ records the estimates of the residual variances for a sample size of $N=100$, with $\left(\widetilde{\sigma}_{\varepsilon}^{2}\right)$ and without $\left(\widehat{\sigma}_{\varepsilon}^{2}\right)$ dummies at 5\%: the sampling distributions for $N=50$ at the same settings were similar. As expected $\widetilde{\sigma}_{\varepsilon}^{2}$ is downwards biased when impulses are introduced. Table 2 reports the average Monte Carlo estimates of $\sigma_{\varepsilon}^{2}$ at $\alpha=0.01$. Since $\sigma_{\varepsilon}^{2}=1$, the expected downward biases in $\widetilde{\sigma}_{\varepsilon}^{2}$ are close to the values of $\left(-2 c_{\alpha} \phi\left(c_{\alpha}\right)\right) \sigma_{\varepsilon}^{2}$ obtained in section 3.2 of -0.066 for $N=50$ and -0.079 for $N=100$. Hence, as the sample size increases, $\widetilde{\sigma}_{\varepsilon}^{2}$ is closer to the relevant limiting value. 


\begin{tabular}{|l|l|l|}
\hline$N$ & $\widehat{\sigma}^{2}$ & $\widetilde{\sigma}^{2}$ \\
\hline 50 & 0.977 & 0.901 \\
100 & 0.989 & 0.910 \\
\hline
\end{tabular}

Table 2: Average across MC replications for $N=50$ and $N=100$

\subsection{Response surface for $\sigma_{\widetilde{\mu}}^{2}$ for normal errors}

The distributional result in section 3 was that:

$$
N^{1 / 2}(\widetilde{\mu}-\mu) \stackrel{D}{\rightarrow} \mathrm{N}\left[0, \sigma_{\epsilon}^{2} \sigma_{\mu}^{2}\right],
$$

so for normal errors when $\lambda_{1}=\lambda_{2}$ from (8):

$$
\sigma_{\mu}^{2}=\frac{1}{(1-\alpha)}\left(1+4 c_{\alpha} \phi\left(c_{\alpha}\right)-\frac{2 c_{\alpha} \phi\left(c_{\alpha}\right)}{(1-\alpha)}\left[1+2 c_{\alpha} \phi\left(c_{\alpha}\right)\right]\right)
$$

and:

$$
\left(\frac{N \operatorname{Var}[\widetilde{\mu}]}{\sigma_{\epsilon}^{2}}\right)=\sigma_{\mu}^{2}
$$

Thus, the simulations generated the values of the left-hand side of (28) which were then regressed on the numerical values of $\sigma_{\mu}^{2}$ computed using (8).

The Monte Carlo simulation first confirmed the invariance of the outcomes from PcGets to the value of $\sigma_{\epsilon}^{2}$ and to the form of 'split' into equal blocks of $m=2$ and $m=3$. There were 78 experiments spanning $c_{\alpha}=5$ to $c_{\alpha}=1\left(\Phi\left(c_{\alpha}\right) \simeq 1\right.$ to $\left.\Phi\left(c_{\alpha}\right)=0.68\right)$ and $N=20$ to $N=300$. The response surface for $\operatorname{Var}[\widetilde{\mu}]$ yielded (HCSE in parentheses: see White, 1980):

$$
\begin{aligned}
\widehat{\operatorname{Var}[\widetilde{\mu}]} & =\underset{(0.0021)}{1.002} N^{-1} \sigma_{\epsilon}^{2} \sigma_{\mu}^{2} \\
\mathrm{R}^{2} & =0.9997 \widehat{\sigma}=1.4 \% \quad \chi_{\text {nd }}^{2}(2)=16.4^{* *} \mathrm{~F}_{\text {het }}(2,75)=21.7^{* *}
\end{aligned}
$$

Some outliers were detected and slightly alter the outcome, but as figure 2a shows, the fitted and actual values are extremely close across the 78 experiments. We also tested for whether the outcome depended on the split being in halves or in thirds and found the corresponding dummy was insignificant.

The outcome using a scaled log form was similar, reported here including the outlier correction for experiments 71-73:

$$
\begin{aligned}
\log \left(\frac{\widehat{N \operatorname{Var}[\widetilde{\mu}]}}{\sigma_{\varepsilon}^{2}}\right) & =\underset{(0.002)}{0.0135}+\underset{(0.011)}{0.936} \log \left(\sigma_{\mu}^{2}\right)+\underset{(0.006)}{0.04} I_{71-73} \\
\mathrm{R}^{2} & =0.9899 \widehat{\sigma}=1.04 \%
\end{aligned}
$$

although all the mis-specification tests were again highly significant.

Figure $2 \mathrm{~b}$ shows the fitted and actual values of (31) across the 78 experiments, and the residuals with their density ( $c$ and $d$ respectively). The fit is extremely close.

\subsection{Non-normality}

We briefly consider the impact of saturation in a highly non-normal case, namely a t(4)-distributed error. Although this distribution does not satisfy the assumptions of the main theorem, it was of interest to see if 'fat-tails' led to an excess of retained impulses. 

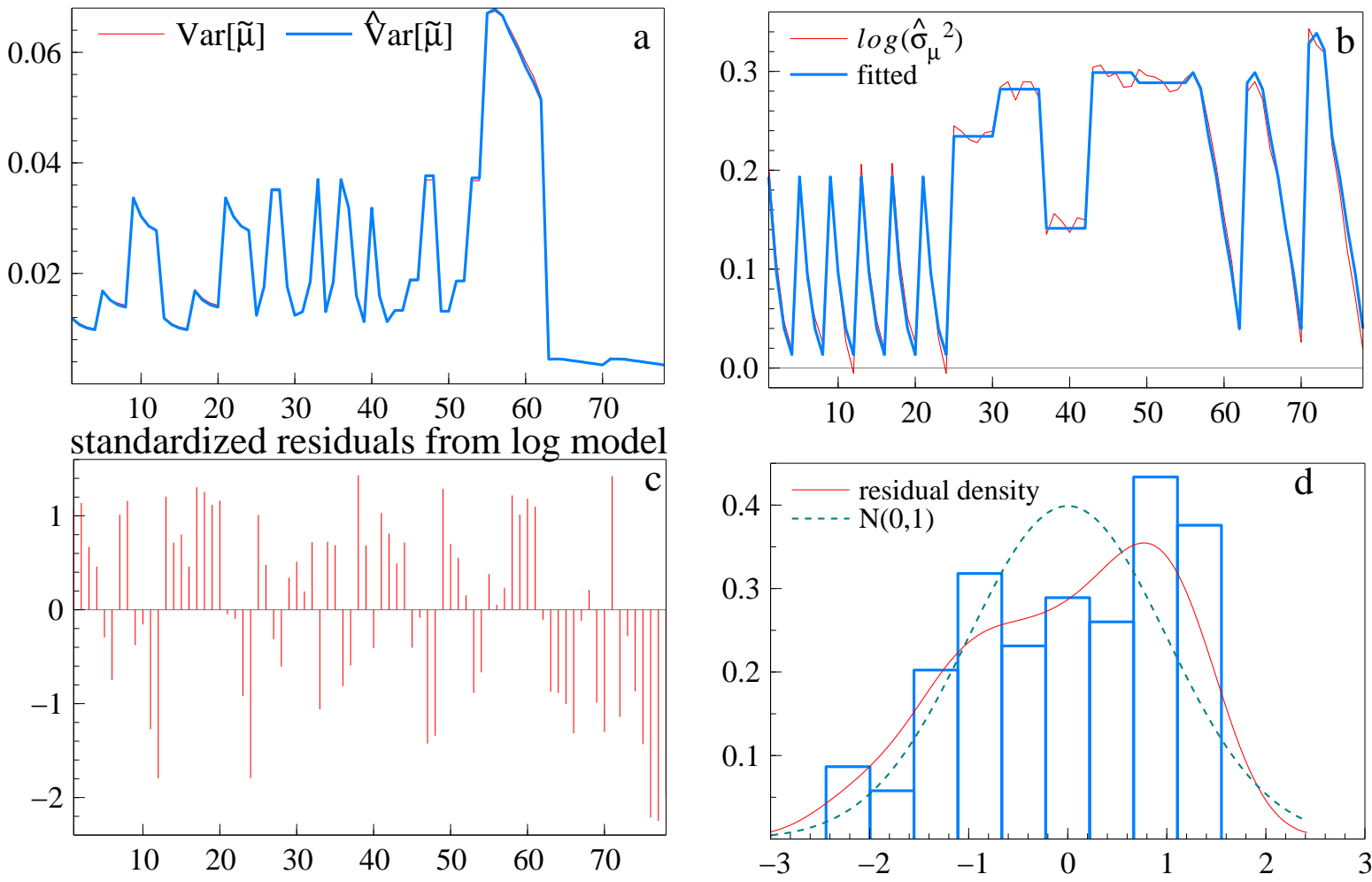

Figure 2: Fitted and actual values from the simulation

A sample size of $N=300$ was considered, for a sample split of $N / 2$. At each replication, the $N$ draws are from a $\mathrm{t}(4)$ distribution. From Johnson, Kotz and Balakrishnan (1995), the moments of $X \sim \mathrm{t}(4)$ are such that $\mathrm{E}(X)=0$ and $\operatorname{Var}(X)=v /(v-2)=2$ where $v$ denotes the degrees of freedom. Hence, when no impulses are added, $\operatorname{Var}(\bar{X})=2 / 300=0.0067$ and $\sqrt{ } \operatorname{Var}(\bar{X})=0.082$.

We use the location-scale DGP in (22) with a $t(4)$ error, but consider two criteria for retention of any single impulse indicator, namely either $\left|\mathrm{t}_{\delta_{i}}\right|>2$ or 2.5 .

Table 3 reports summary statistics from the Monte Carlo experiments, where ARNI stands for the average number of retained impulses in each replication. There is little evidence of an excess retention of impulses. The intuitive explanation is that the fat tails generate a much larger residual error variance, so only draws far into the tails are significant even though a nominal critical value relevant to the normal is used.

\begin{tabular}{|l|l|l|}
\hline$N=300$ & $\left|\mathrm{t}_{\delta_{i}}\right|>2$ & $\left|\mathrm{t}_{\delta_{i}}\right|>2.5$ \\
\hline $\mathrm{E}[\widetilde{\mu}]$ & -0.002 & -0.008 \\
$\operatorname{Var}(\widetilde{\mu})$ & 0.00544 & 0.00535 \\
\hline ARNI & 15.64 & 8.08 \\
$\mathrm{RF}$ & $5.2 \%$ & $2.7 \%$ \\
\hline
\end{tabular}

Table 3: Results for an N/2 split drawing the errors from a $\mathrm{t}(4)$

\section{Power}

Naturally, the power of the procedure to detect any form of break depends on the nature and magnitude of the departure from the null. Two cases of interest are a mixture of distributions with considerably 
different variances, where the indicators will 'select' mainly observations drawn from the high variance distribution; and location shifts, where a subset of the sample is drawn with a different mean.

As a specific example, consider when $\mu$ takes two values $\mu$ and $\mu^{*}$, pre and post an observation $N^{*}$ say. Providing the selected sub-samples include indicators covering all of the break and 'outside break' observations, then blocks of $d_{i, j}$ will be significant with an average value equal to $\left(\mu-\mu^{*}\right)$, and thereby reveal a step shift. As noted above, conditional on the retained $\delta_{i}$, tests for combinations do not alter the null rejection frequency. However, outlier detection algorithms can fail to detect any problem in that setting, since the overall sample mean is the value that balances mean deviations, and if both groups, pre and post break, are a substantial proportion of $N$, then the large induced value of the estimated residual standard deviation will include almost every outcome as figure 3 illustrates.
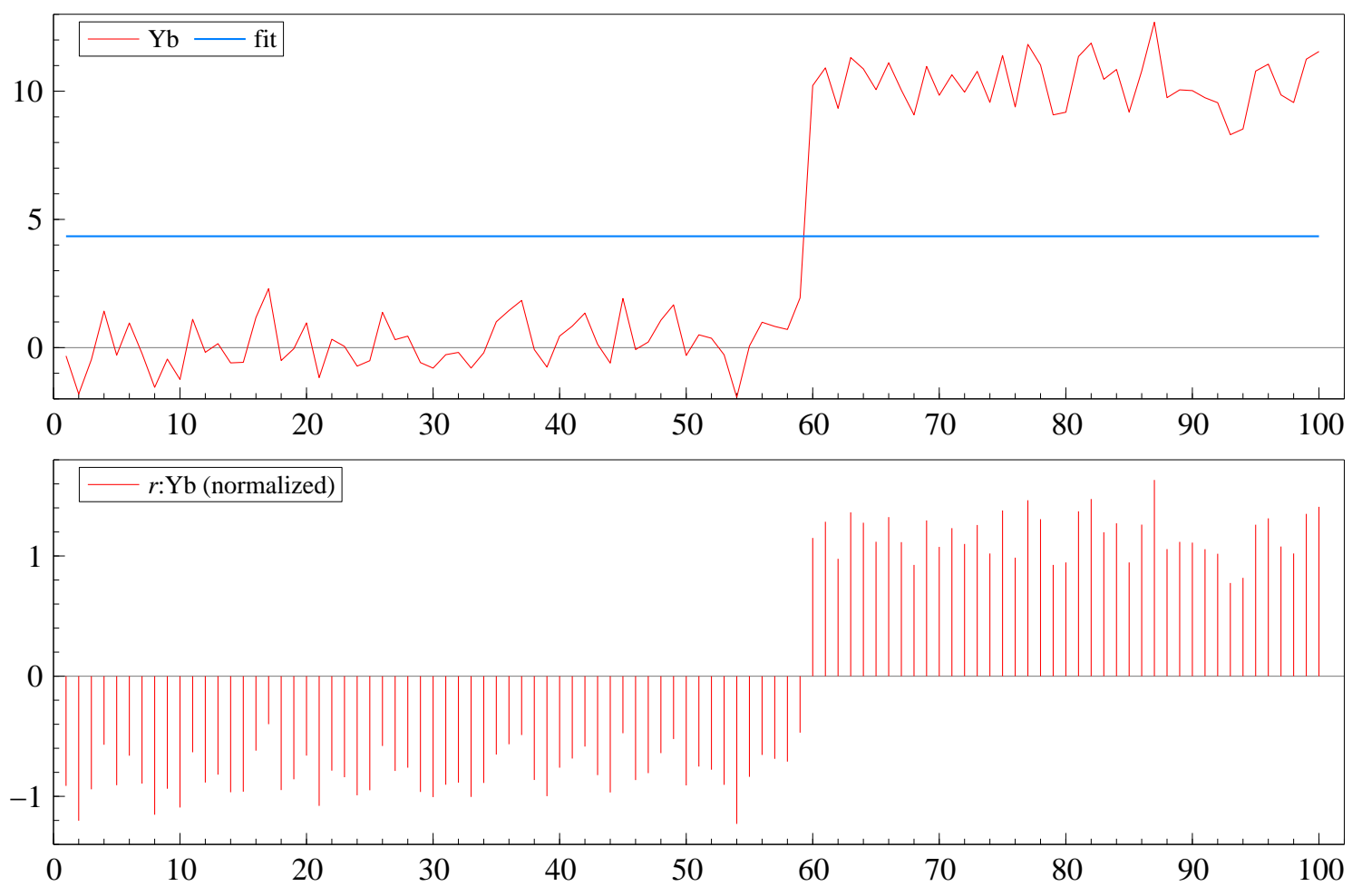

Figure 3: Absence of outliers despite a break

The procedure we propose could also reveal model mis-specification. For example, in a time-series context, consider a model where $y_{t-1}$ has been included as a regressor, despite being irrelevant, when there were no indicators but a mean shift occurred as in figure 3. Then its coefficient would reflect the step shift and would be close to unity, thereby removing the mean shift except at its end points where impulses of roughly equal magnitude, opposite sign would be created: see e.g., Perron (1989), and Hendry and Neale (1991). A conventional 'outlier removal' approach would again conclude with the incorrect model, albeit one which may be viable for forecasting. Adding the blocks of indicators, in this simple case, would clarify that there is a step shift, but no dynamics. Thus, there are clear uses for such a 'saturation' approach.

\section{Conclusion}

We have considered a problem that previously seemed intractable: selecting a regression when there are more regressors than observations. The special case we examined was for saturating the model 
with individual impulse indicators, one for each observation. A variant of the general-to-simple (Gets) approach nevertheless suggested a feasible solution. Aspects of the distributions of the mean, its standard error, and the residual standard deviation, after retaining only significant impulses from the saturating set, were derived, together with an approximate operational bias correction for the last of these.

To select a regression when there are more regressors than observations requires both a block implementation of multi-path searches, as well as such procedures within tentative models as in PcGets. The Monte Carlo simulations based on doing so match the theoretical analysis, confirming that the approach is viable, with the null rejection frequencies as established above.

Moreover, many new problems become amenable to solution, including general regression sub-set selection, non-linear model selection, and new automatically computable tests of economic interest (see Hendry and Santos, 2006).

\section{References}

Campos, J., Ericsson, N. R., and Hendry, D. F. (2004). Editors' introduction. In Campos, J., Ericsson, N. R., and Hendry, D. F. (eds.), Readings on General-to-Specific Modeling, pp. 1-81. Cheltenham: Edward Elgar.

Campos, J., Hendry, D. F., and Krolzig, H.-M. (2003). Consistent model selection by an automatic Gets approach. Oxford Bulletin of Economics and Statistics, 65, 803-819.

Doornik, J. A. (1999). Object-Oriented Matrix Programming using Ox. London: Timberlake Consultants Press. 3rd edition.

Foster, D. P., and Stine, R. A. (2004). Honest confidence intervals for the error variance in stepwise regression. Mimeo, Statistics Department, Wharton School, University of Pennsylvania.

Granger, C. W. J., and Hendry, D. F. (2005). A dialogue concerning a new instrument for econometric modeling. Econometric Theory, 21, 278-297.

Hendry, D. F. (1995). Dynamic Econometrics. Oxford: Oxford University Press.

Hendry, D. F., and Krolzig, H.-M. (2001). Automatic Econometric Model Selection. London: Timberlake Consultants Press.

Hendry, D. F., and Krolzig, H.-M. (2003). New developments in automatic general-to-specific modelling. In Stigum, B. P. (ed.), Econometrics and the Philosophy of Economics, pp. 379-419. Princeton: Princeton University Press.

Hendry, D. F., and Krolzig, H.-M. (2004). Model selection with more variables than observations. Unpublished paper, Economics Department, Oxford University.

Hendry, D. F., and Krolzig, H.-M. (2005). The properties of automatic Gets modelling. Economic Journal, 115, C32-C61.

Hendry, D. F., and Neale, A. J. (1991). A Monte Carlo study of the effects of structural breaks on tests for unit roots. In Hackl, P., and Westlund, A. H. (eds.), Economic Structural Change, Analysis and Forecasting, pp. 95-119. Berlin: Springer-Verlag.

Hendry, D. F., and Santos, C. (2005). Regression models with data-based indicator variables. Oxford Bulletin of Economics and Statistics, 67, 571-595.

Hendry, D. F., and Santos, C. (2006). Automatic tests of super exogeneity. Unpublished paper, Economics Department, University of Oxford.

Hoover, K. D., and Perez, S. J. (1999). Data mining reconsidered: Encompassing and the general-tospecific approach to specification search. Econometrics Journal, 2, 167-191. 
Hoover, K. D., and Perez, S. J. (2004). Truth and robustness in cross-country growth regressions. Oxford Bulletin of Economics and Statistics, 66, 765-798.

Johnson, N. L., Kotz, S., and Balakrishnan, N. (1995). Continuous Univariate Distributions - 2 2nd edn. New York: John Wiley.

Krolzig, H.-M., and Hendry, D. F. (2001). Computer automation of general-to-specific model selection procedures. Journal of Economic Dynamics and Control, 25, 831-866.

Perron, P. (1989). The Great Crash, the oil price shock and the unit root hypothesis. Econometrica, 57, 1361-1401.

Salkever, D. S. (1976). The use of dummy variables to compute predictions, prediction errors and confidence intervals. Journal of Econometrics, 4, 393-397.

White, H. (1980). A heteroskedastic-consistent covariance matrix estimator and a direct test for heteroskedasticity. Econometrica, 48, 817-838. 